\title{
Editorial: Blindness in two worlds
}

In this issue we publish three surveys of blindness, one from the Gambia and two from the United Kingdom. As might be expected there are great differences between the two. Strict comparison is difficult because the African and UK studies were carried out differently, the former being a prospective clinical study of actual patients in a sample of the population but the latter an examination of Blind Certificates. Nevertheless in spite of this difference it is still possible to perceive dissimilarities between the pattern of blindness in Africa and in the UK.

The two principal features of interest in such surveys are, first, the number of blind persons as a proportion of the population (the 'burden of blindness'), and secondly the causes of the blindness. The Gambia differs from the UK in both respects.

So far as the Gambia is concerned the prevalence of blindness is estimated to be $0.7 \%$ of the population (of 800000). Unfortunately it is not possible to compare this with anything in the present UK figures, since they are quoted in terms of annual incidence of blind registration, but it is known from other studies that the prevalence of blindness in the UK is $0 \cdot 18 \%$. $^{1}$ When it is considered that the average age of the population in the Gambia is probably lower than that of the UK (though this figure is not quoted in the present papers) the difference becomes even more emphatic. It is not without significance, one would think, that the periurban area of Banjul in the Gambia showed much less visual impairment than the poorer slum area of the capital, where the prevalence was similar to that in the rural areas. One would expect that the principal causes of differences between the Third World and the UK would rest on economic grounds, so that it is interesting to have this internal confirmation.

The principal causes of blindness in the Gambia in numerical order are cataract and uncorrected aphakia $55 \%$, non-trachomatous corneal opacity and phthisis bulbi $20 \%$, trachoma $17 \%$, and all other causes $8 \%$. Glaucoma accounted for only $2 \%$, but the authors believe this may be an underestimate, since their study did not include visual fields.

It is noticeable that macular degeneration makes no impact whatsoever on the blind statistics of the African study and that the overwhelming burden comes from cataract and corneal disease. It is probable that as antibiotics gradually become more available (one hopes) that the burden of corneal blindness will be reduced in time. Unfortunately the same cannot be said for cataract, and although there have been useful recent investigations into the possible causes of cataract, linking it in particular to diarrhoea, ${ }^{2}$ there is as yet no real prospect of preventing it. Thus until more surgery can be delivered to the underdeveloped countries, there is no hope of the cataract burden being reduced.

Compared with the Gambia we in the UK are in clover. Our cataract burden has been reduced to an astonishing degree, not only in contrast to the state of affairs in Africa but also in 1985 compared with 1959.

There is one particularly disappointing statistic, however, and this concerns glaucoma. In Table 11 of the Avon study it can be seen that the 'all ages' incidence rate per 100000 population has worsened between 1959 and 1985 from $2 \cdot 60$ to $4 \cdot 70$. However, the age-banded rate for $60-69$ shows an improvement from $5 \cdot 20$ to $2 \cdot 80$, whereas the rate for the $70+$ group shows a deterioration from $30 \cdot 90$ to $37 \cdot 0$. The 'all ages' rates may simply be reflecting a change in the age make-up of the population-an increasing number of old people producing an increasing number of cases of glaucomatous blindness.

However, the statistics from Leicester show an increase in blindness from glaucoma in the age groups 65 and over during the last 20 years, from 18 per 100000 in 1965 to 29 per 100000 in 1985 . Thus when both sets of UK statistics are taken together it remains uncertain whether there is any actual change in the incidence of blindness from glaucoma.

REDMOND J H SMITH

\section{References}

1 Department of Health and Social Security (Registered Blind and Partially Sighted persons) England 1979. In report of World Health Organisation. Available data in blindness (update 1987).

2 Minassian WC, Mehra V, Jones BR. Dehydrational crises from severe diarrhoea or heatstroke and risk of cataract. Lancet 1984; i: 751-3. 